\title{
Excesso de peso em mulheres de uma comunidade urbana de baixa renda: fatores socioeconômicos, demográficos e reprodutivos
}

\author{
Excess weight among women in a low-income urban community: \\ socioeconomic, demographic and reproductive factors
}

Letícia Dinegri (https://orcid.org/0000-0002-8890-3727) ${ }^{1}$

Malaquias Batista Filho (https://orcid.org/0000-0002-1490-0590) ${ }^{2}$

Helânia Virginia Dantas dos Santos (https://orcid.org/0000-0003-2562-6029) ${ }^{1}$

Pedro Israel Cabral de Lira (https://orcid.org/0000-0002-1534-1620) ${ }^{3}$

Poliana Coelho Cabral (https://orcid.org/0000-0002-2709-4823) ${ }^{3}$

Sophie Helena Eickmann (https://orcid.org/0000-0002-6523-2107) ${ }^{4}$

Marilia de Carvalho Lima (https://orcid.org/0000-0002-4993-2584) ${ }^{4}$

${ }^{1}$ Programa de Pós-

Graduação em Nutrição,

Universidade Federal de

Pernambuco (UFPE). Av.

Prof. Moraes Rego 1235,

Cidade Universitária. 50670-

901 Recife PE Brasil.

letydinegri@yahoo.com.br

${ }^{2}$ Instituto de Medicina

Integral Professor Fernando

Figueira. Recife PE Brasil.

${ }^{3}$ Centro de Ciências da

Saúde, Departamento de

Nutrição, UFPE. Recife PE

Brasil.

${ }^{4}$ Programa Pós-Graduação

em Saúde da Criança e do

Adolescente, UFPE. Recife

PE Brasil.

\begin{abstract}
The aim of the present study was to investigate the prevalence of excess weight and associated factors in women of reproductive age living in a low-income community. A cross-sectional study was conducted with a sample of 663 women 15 to 49 years of age residing in the neighborhood of Coelhos in the city of Recife, Brazil. Body mass index (BMI)-for-age was used to classify the nutritional status of the adolescents (15 to 19 years of age), adopting $Z$-score of $\geq+1$ for the definition of overweight. For the adults, $B M I \geq 25.0$ $\mathrm{kg} / \mathrm{m}^{2}$ was considered indicative of overweight. Socioeconomic, demographic and reproductive variables were analyzed as possible factors associated with overweight. The prevalence of excess weight was found in two thirds of the sample. The results of the Poisson multiple regression analysis showed a significantly higher prevalence of excess weight with the advance in age, among those with a younger menarche age, those who had three or more pregnancies, those living with their partner and those self-declared black or white. Multiparity was the only factor associated with excess weight that could be modified, which underscores the importance of prenatal and family planning services to its prevention and control.
\end{abstract}

Key words Poverty areas, Body Mass Index, Overweight, Menarche, Pregnancy
Resumo O objetivo do estudo foi avaliar a prevalência de excesso de peso e fatores associados nas mulheres em idade reprodutiva, residentes em uma comunidade de baixa renda. Estudo transversal cuja amostra consistiu em 663 mulheres, na faixa etária de 15 a 49 anos, domiciliadas na comunidade dos Coelhos, Recife-PE. O IMC para idade foi utilizado para classificar o estado nutricional de mulheres entre 15 e 19 anos, considerando $\geq+1$ escore $Z$ para definir excesso de peso. Nas mulheres com idade superior a 19 anos, considerou-se o IMC $\geq 25,0 \mathrm{~kg} / \mathrm{m}^{2}$. Variáveis socioeconômicas, demográficas e reprodutivas foram analisadas como possiveis fatores associados ao excesso de peso. A prevalência do excesso de peso foi observada em dois terços das mulheres estudadas. Os resultados da análise de regressão múltipla de Poisson mostraram uma prevalência significantemente maior do excesso de peso em mulheres com o avançar da idade cronológica, com menor idade da menarca, que tiveram três ou mais gestações, que coabitavam com o companheiro e se autodenominaram com cor preta ou cor branca. A multiparidade foi o único fator associado ao excesso de peso passivel de modificação, o que reforça a necessidade de destacar a importância dos serviços de pré-natal e planejamento familiar na sua prevenção e controle.

Palavras-chave Áreas de pobreza, Índice de Massa Corporal, Sobrepeso, Menarca, Número de gestações 


\section{Introdução}

O excesso de peso é um problema de saúde pública que afeta uma grande parcela da população adulta em todo o mundo ${ }^{1,2}$, e um percentual significativo das mulheres em idade reprodutiva ${ }^{1,3-5}$. Ao comparar os dados do Estudo Nacional de Despesa Familiar (1974-1975), primeiro inquérito brasileiro sobre consumo alimentar, estrutura de despesa familiar e estado nutricional de uma amostra representativa da população com dados da Vigilância de Fatores de Risco e Proteção para Doenças Crônicas (2017), o excesso de peso em mulheres duplicou, elevando-se de $28,7 \%$ para $51,2 \%{ }^{4,5}$.

O excesso de peso é uma doença multifatorial, secundária ao aumento na ingestão de alimentos e redução do gasto calórico. Entre os fatores que desencadeiam esse processo incluem-se os genéticos, biológicos, sociais e ambientais, entre estes se destacam mudanças inadequadas no padrão alimentar e redução da atividade física ${ }^{6-10}$. Levando-se em consideração os fatores biológicos, relacionados ao ciclo reprodutivo feminino, ressaltam-se a idade da menarca e a paridade ${ }^{10-14}$.

No âmbito socioeconômico e demográfico, poucos estudos têm sido conduzidos no sentido de analisar o aumento do excesso de peso no contexto da pobreza, sobretudo em mulheres domiciliadas em comunidades de baixa renda, as quais têm sido mais vulneráveis aos determinantes sociais da variação do peso ${ }^{15-17}$. Frente ao panorama de iniquidades sociais tem sido levantada a hipótese de que o ganho de peso é decorrente de condições insuficientes para manutenção de consumo alimentar saudável ${ }^{17}$. Assim sendo, a hipótese que englobaria todos os contextos nos quais a mulher está inserida, envolve a associação das condições socioeconômicas, ambientais e biológicas desfavoráveis, cuja interrelação condiciona o excesso de peso.

Portanto, o presente estudo tem por objetivo avaliar a prevalência de excesso de peso e fatores associados em mulheres em idade reprodutiva, residentes em uma comunidade de baixa renda na Região Metropolitana do Recife.

\section{Métodos}

Estudo transversal realizado com mulheres em idade reprodutiva residentes na comunidade dos Coelhos, Recife, Pernambuco, tendo por base os dados da pesquisa "Saúde, nutrição e serviços assistenciais numa população favelada do Recife: um estudo baseline". Esta comunidade tem uma população de aproximadamente 7.633 habitantes, residentes em 2.322 domicílios, com rendimento nominal médio mensal das habitações de R\$ 898,41, com proporção de 53,6\% dos domicílios chefiados por mulheres, taxa de alfabetização de $87,7 \%$ na população com 10 ou mais anos de idade e densidade média de 3,6 moradores por domicílio ${ }^{18}$. A assistência de saúde é prestada por duas Unidades de Saúde da Família.

O estudo utilizou técnica de amostragem do tipo aleatória simples sem reposição. Para garantir a representatividade da amostra, os domicílios foram selecionados por sorteio aleatório com probabilidade proporcional à estimativa do número total de mulheres na faixa etária entre $15 \mathrm{e}$ 49 anos residentes no local de estudo.

O cálculo do tamanho da amostra para a prevalência de excesso de peso, baseou-se em estudo anterior ${ }^{6}$ que obteve prevalência de $41,7 \%$ de excesso de peso em mulheres em idade reprodutiva. Estabelecendo-se uma margem de erro amostral máxima de 5,0\% e intervalo de confiança de 95,0\%, estimou-se um tamanho amostral de 374 mulheres. Para estimar o tamanho da amostra na investigação dos fatores associados ao excesso de peso, tomamos como base a idade da menarca como uma das variáveis independentes de interesse para o estudo. Adotando-se os dados obtidos por Pinheiro et al. ${ }^{19}$ que encontraram percentual de $54,0 \%$ de excesso de peso em mulheres de 15 a 49 anos com menarca acima dos 13 anos e $67,7 \%$ de excesso de peso entre as que tiveram menarca com 13 anos ou menos de idade, um poder do estudo de $80,0 \%$, intervalo de confiança de 95,0\% e razão de 1:3 entre expostos: não expostos obtivemos uma estimativa amostral total de 572 mulheres (143 expostas:429 não expostas). Ao se considerar possíveis perdas amostrais acrescentou-se $16,0 \%$ à estimativa, resultando em uma amostra mínima de 663 mulheres.

Os critérios de elegibilidade para inclusão das mulheres no estudo foram: idade entre 15 e 49 anos e que residissem na comunidade dos Coelhos. Foram excluídas as mulheres que, no momento da coleta de dados, não tivessem apresentado a menarca, encontravam-se gestantes, e aquelas que possuíssem incapacidade cognitiva para responder ao estudo ou alguma limitação física que impedisse a avaliação antropométrica.

A variável dependente foi o estado nutricional das mulheres, avaliado pelo Índice de Massa Corporal por Idade (IMC/I) nas adolescentes e pelo IMC nas adultas. As variáveis independentes foram representadas de forma categórica, in- 
cluindo: classe econômica (B1/B2/C1; C2; D/E); faixa etária $(15-19 ; 20-35 ; 36-49$ anos); cor da pele (parda, amarela e indígena; preta; branca); escolaridade $(\leq 8 ; \geq 9$ anos de estudo); situação conjugal (sem e com companheiro); trabalho formal (não; sim); chefe da família (não; sim); beneficiária do Programa Bolsa Família (não; sim); idade da menarca $(<12 ; \geq 12$ anos); número de gestações (nulípara; $1-2 ; \geq 3$ ); triagem dos transtornos mentais comuns $(<8$ pontos=não; $\geq 8$ pontos $=\operatorname{sim}$ ), conforme classificação de Mari e Williams ${ }^{20}$.

A coleta das informações da família e aferição antropométrica foram realizadas nos domicílios no período de junho a dezembro de 2014, por equipe de trabalho de campo treinada. Para medida de peso foi utilizada balança eletrônica (Modelo MEA-03200/Plenna) com capacidade de $150 \mathrm{~kg}$ e sensibilidade de $100 \mathrm{~g}$. A altura foi aferida através de estadiômetro portátil (Alturaexata ${ }^{\circledR}$, Ltda) - milimetrado, com $213 \mathrm{~cm}$ e precisão de $1 \mathrm{~mm}$. As medidas de peso e altura foram mensuradas segundo técnicas preconizadas por Lohman et al. ${ }^{21}$, aferidas em duplicata e de forma sequencial, sendo utilizada a média desses valores. Para consistência dos dados, foram repetidas as medidas que apresentassem diferenças superiores a $100 \mathrm{~g}$ para o peso e $0,5 \mathrm{~cm}$ para altura, sendo utilizada a média dos dois valores mais próximos.

O estado nutricional das adolescentes foi avaliado pelo Índice de Massa Corporal/Idade expresso em escore $\mathrm{z}$, utilizando-se o programa AnthroPlus versão 3.2.2. Foram considerados como referência, os pontos de corte estabelecidos pela $\mathrm{WHO}^{22}$ : baixo peso $(<-2$ escores $\mathrm{z})$, eutrofia $(\geq-2$ e $<+1$ escore $z)$, sobrepeso $(\geq+1$ e $<+2$ escores $z)$ e obesidade ( $\geq+2$ escores $z$ ). Para a análise estatística foram criadas duas categorias: sem excesso de peso, representada pela soma dos valores do baixo peso e da eutrofia, e com excesso de peso, representado pela soma dos valores do sobrepeso e da obesidade.

A avaliação do estado nutricional das mulheres com idade igual ou superior a 20 anos foi realizada através do IMC, segundo a classificação da $\mathrm{WHO}^{23}$. Foram considerados os seguintes pontos de corte para o IMC: baixo peso $\left(<18,5 \mathrm{~kg} / \mathrm{m}^{2}\right)$, eutrofia $\left(18,5\right.$ e $\left.24,9 \mathrm{~kg} / \mathrm{m}^{2}\right)$, sobrepeso $(25,0$ e $\left.29,9 \mathrm{~kg} / \mathrm{m}^{2}\right)$ e obesidade $\left(\geq 30,0 \mathrm{~kg} / \mathrm{m}^{2}\right)$. Para a análise estatística foram criadas duas categorias, semelhantes às utilizadas na avaliação do estado nutricional das adolescentes.
Para estratificação da classe econômica das famílias utilizou-se o Critério de Classificação Econômica do Brasil (CCEB) da Associação Brasileira de Empresas de Pesquisa $(\mathrm{ABEP})^{24}$. Esta classificação possui um sistema de atribuição de pontos, em função da posse de bens domésticos e do grau de instrução do chefe da família que, através da soma destes pontos, estima as classes econômicas definidas como A1, A2, B1, B2, C1, C2, D, E.

A triagem para transtornos mentais comuns foi realizada através do Self-Reporting Questionnaire (SQR-20), desenvolvido pela $\mathrm{WHO}^{25}$ e validado para a população brasileira ${ }^{20}$. Este instrumento é composto de 20 questões, com escore total que pode variar de 0 a 20 pontos, com valores mais elevados indicando maior probabilidade de apresentar traços de depressão e ansiedade.

Os questionários elaborados com perguntas fechadas e pré-codificadas eram revisados regularmente durante a coleta de dados. Os dados foram digitados em dupla entrada, com a finalidade de verificar a consistência da digitação, através do comando Data Compare no Epi-Info 3.5.4 para Windows.

As análises bivariadas foram realizadas no Statistical Package for the Social Sciences, versão 13.0 (SPSS Inc., Chicago, EUA), a fim de verificar a associação entre as variáveis independentes e o desfecho, através do teste Qui-quadrado de Pearson. As variáveis que apresentaram valor $\mathrm{p}<0,20$ nas análises bivariadas foram selecionadas para inclusão na análise de Regressão Múltipla de Poisson.

O cálculo das razões de prevalência (RP) brutas e ajustadas e os respectivos intervalos de 95\% de confiança foram obtidos pela Regressão de Poisson, utilizando o software STATA 7.0 (Stata, Texas, EUA). A entrada das variáveis independentes nos modelos de regressão foi realizada em blocos, seguindo um modelo conceitual hierarquizado previamente estabelecido. Inicialmente foram introduzidas as variáveis do bloco I: classe econômica, idade, cor da pele e situação conjugal. Por último, no segundo bloco foi adicionada a variável idade da menarca e número de gestações.

O presente estudo foi aprovado no Comitê de Ética em Pesquisa do Instituto de Medicina Integral Professor Fernando Figueira (IMIP). O Termo de Consentimento Livre e Esclarecido (TCLE) ${ }^{26}$ foi assinado por todas as participantes acima dos 18 anos e pelos representantes legais das participantes com idade inferior a 18 anos. 


\section{Resultados}

A Tabela 1 apresenta a classificação do estado nutricional das mulheres avaliado através do IMC. O excesso de peso foi identificado em dois terços das mulheres estudadas $(66,3 \%)$, sendo a proporção de sobrepeso discretamente maior $(35,7 \%)$ do que a da obesidade $(30,6 \%)$.

Na Tabela 2 estão apresentadas as características sociodemográficas, reprodutivas e comportamentais da amostra estudada. Mais da metade das mulheres pertencia às classes $\mathrm{B} 1 / \mathrm{B} 2 \mathrm{e} \mathrm{C} 1 / \mathrm{C} 2$ $(66,4 \%)$ e tinham escolaridade acima de oito anos $(64,6 \%)$. Metade da amostra apresentava idade entre 20 e 35 anos (50,5\%), apenas $40,6 \%$ conviviam com o companheiro, $30,6 \%$ eram chefe da família e 62,0\% eram beneficiárias do PBF. Os percentuais de mulheres com excesso de peso foram significantemente mais elevados com o progredir da idade cronológica, entre as que se autodeclararam pretas ou brancas, as que viviam com o companheiro, referiram idade da menarca antes dos 12 anos e tiveram três ou mais gestações.

A Tabela 3 apresenta o resultado das análises de Regressão de Poisson simples e múltipla dos fatores sociodemográficos e reprodutivos associados ao excesso de peso em mulheres. As Razões de Prevalência (RP) ajustadas foram significantemente maiores com o avançar da idade cronológica, entre as mulheres que se autodeclararam pretas e brancas, que conviviam com o companheiro, referiram ocorrência da menarca antes dos 12 anos e tiveram três ou mais gestações.

\section{Discussão}

Essa pesquisa, conduzida em uma comunidade de baixa renda na cidade do Recife, investigou a prevalência e os fatores associados ao excesso de peso em mulheres em idade fértil. Os resultados indicaram que dois terços das mulheres residentes nesta comunidade apresentaram esse desfecho, tendo como fatores associados, a idade cronológica, cor da pele, situação conjugal, a idade quando do primeiro episódio menstrual e o número de gestações.

A elevada prevalência de excesso de peso em mulheres revelada no presente estudo, reflete que esse é um problema na população feminina, conforme dados publicados pela $\mathrm{WHO}^{1}$. Outros dados da literatura corroboram com estes resultados e dão robustez ao nosso achado ${ }^{27-29}$.

A elevada magnitude do excesso de peso na população estudada reforça a ocorrência da epi- demia da obesidade, presente nos países emergentes, a exemplo do Brasil. Essa tendência de crescimento também foi evidenciada em duas pesquisas de base populacional, realizadas no estado de Pernambuco, nos anos de 1997 e $2006^{6}$. O mesmo foi observado em nível nacional, através do Inquérito Telefônico Brasileiro (VIGITEL) realizado na população feminina nas capitais brasileiras, com percentuais de $38,8 \%$ em $2006^{29}$ e $51,2 \%$ em $2017^{5}$. A prevalência de excesso de peso em mulheres tem aumentado em países desenvolvidos, como nos Estados Unidos $(63,2 \%)$, no Reino Unido $(58,9 \%)$ e Canadá $(58,5 \%)^{3}$, e em desenvolvimento, como o Chile $(61 \%)^{30}$ e a Jamaica $(62 \%)^{31}$.

Dentre os fatores associados ao excesso de peso, a variável idade se destaca, observando-se aumento da prevalência com a sua progressão. Resultados semelhantes têm sido descritos em outros estudos ${ }^{12,32,33}$. A elevada prevalência de excesso de peso em mulheres mais velhas, ocorre provavelmente devido a uma maior deposição de gordura ao longo dos anos, quando se observa uma redução do metabolismo basal ${ }^{34}$ e um decréscimo do nível de atividade física.

A cor da pele, branca e negra, também se associou ao excesso de peso. Este achado se apresenta contraditório com os da literatura, evidenciado no inquérito telefônico realizado com população de adultos e idosos, que apontou para uma maior suscetibilidade ao excesso de peso apenas nas mulheres negras ${ }^{35}$. Por outro lado, dados de pesquisa realizada no Nordeste brasileiro ${ }^{6}$, não revelou associação significante do excesso de peso com a cor da pele. A discrepância entre esses resultados, pode ser devido ao fato de a variável cor da pele ter sido autorreferida, o que pode ter favorecido a ocorrência de erro de classificação.

Conviver com o companheiro foi outra variável demográfica que influenciou o excesso de

Tabela 1. Estado nutricional de mulheres dos 15 aos 49 anos. Recife, Brasil, 2017.

\begin{tabular}{lcr}
\hline \multicolumn{1}{c}{ Estado Nutricional ${ }^{\star}$} & $\mathbf{n = 6 6 3}$ & \multicolumn{1}{c}{$\mathbf{\%}$} \\
\hline Baixo Peso & 21 & 3,2 \\
Eutrofia & 202 & 30,5 \\
Excesso de peso & 440 & 66,3 \\
$\quad$ Sobrepeso & 237 & 35,7 \\
$\quad$ Obesidade & 203 & 30,6 \\
\hline
\end{tabular}

${ }^{\star}$ Estado nutricional avaliado pelo Índice de Massa Corporal por Idade (IMC/I) nas adolescentes (WHO, 2009) e pelo IMC nas adultas (WHO, 1995).

Fonte: Elaborado pelos autores. 
Tabela 2. Excesso de peso em mulheres dos 15 aos 49 anos, segundo condições sociodemográficas, reprodutivas e comportamentais. Recife, Brasil, 2017.

\begin{tabular}{|c|c|c|c|c|c|c|}
\hline \multirow{2}{*}{ Variáveis } & \multicolumn{2}{|c|}{ Total } & \multicolumn{3}{|c|}{ Excesso de peso } & \multirow{2}{*}{ Valor $\mathrm{p}$} \\
\hline & $n=663$ & $\%$ & $\mathbf{n}$ & $\%$ & IC95\% & \\
\hline Classe Econômica & & & & & & 0,150 \\
\hline $\mathrm{D}, \mathrm{E}$ & 223 & 33,6 & 137 & 61,4 & $54,9-67,6$ & \\
\hline $\mathrm{C} 2$ & 270 & 40,7 & 185 & 68,5 & $62,9-74,0$ & \\
\hline $\mathrm{B} 1, \mathrm{~B} 2, \mathrm{C} 1$ & 170 & 25,7 & 118 & 69,4 & $62,4-76,4$ & \\
\hline Faixa etária (anos) & & & & & & $<0,001$ \\
\hline $15-19$ & 64 & 9,7 & 25 & 39,1 & $28,1-51,3$ & \\
\hline $20-35$ & 335 & 50,5 & 212 & 63,3 & $58,0-68,2$ & \\
\hline $36-49$ & 264 & 39,8 & 203 & 76,9 & $71,5-81,6$ & \\
\hline Cor da pele & & & & & & $<0,001$ \\
\hline Parda, Amarela e Indígena & 452 & 68,2 & 281 & 62,2 & $57,6-66,6$ & \\
\hline Preta & 76 & 11,5 & 57 & 75,0 & $65,0-84,9$ & \\
\hline Branca & 135 & 20,4 & 102 & 75,6 & $67,7-82,0$ & \\
\hline Escolaridade (anos) & & & & & & 0,858 \\
\hline$\leq 8$ & 235 & 35,4 & 157 & 66,8 & $67,7-82,0$ & \\
\hline$\geq 9$ & 428 & 64,6 & 283 & 66,1 & $61,5-70,4$ & \\
\hline Situação conjugal & & & & & & $<0,001$ \\
\hline Sem companheiro & 394 & 59,4 & 239 & 60,7 & $55,7-65,3$ & \\
\hline Com companheiro & 269 & 40,6 & 201 & 74,7 & $69,3-79,5$ & \\
\hline Trabalho formal & & & & & & 0,581 \\
\hline Não & 323 & 48,7 & 211 & 65,3 & $60,0-70,3$ & \\
\hline Sim & 340 & 51,3 & 229 & 67,4 & $62,3-74,9$ & \\
\hline Chefe da família & & & & & & 0,346 \\
\hline Não & 460 & 60,4 & 300 & 65,2 & $60,8-69,4$ & \\
\hline Sim & 203 & 30,6 & 140 & 69,0 & $62,3-74,9$ & \\
\hline Beneficiária do PBF & & & & & & 0,988 \\
\hline Não & 252 & 38,0 & 162 & 64,3 & $58,2-69,9$ & \\
\hline Sim & 411 & 62,0 & 278 & 67,6 & $63,0-72,0$ & \\
\hline Idade da menarca (anos) & & & & & & 0,033 \\
\hline$\geq 12$ & 515 & 77,7 & 331 & 64,3 & $60,0-68,3$ & \\
\hline$<12$ & 148 & 22,3 & 109 & 73,6 & $66,0-80,0$ & \\
\hline Número de gestações & & & & & & $<0,001$ \\
\hline Nulípara & 93 & 14,0 & 45 & 48,4 & $38,5-58,4$ & \\
\hline $1-2$ & 301 & 45,4 & 188 & 62,5 & $56,9-67,7$ & \\
\hline$\geq 3$ & 269 & 40,6 & 207 & 77,0 & $71,6-81,6$ & \\
\hline Transtornos mentais comuns (SQR-20) & & & & & & 0,278 \\
\hline Não (<8 pontos) & 482 & 72,7 & 314 & 65,1 & $60,8-69,4$ & \\
\hline $\operatorname{Sim}(\geq 8$ pontos $)$ & 181 & 27,3 & 126 & 69,6 & $62,8-76,3$ & \\
\hline
\end{tabular}

IC95\%: Intervalo de 95\% de confiança; PBF: Programa Bolsa Família; SQR-20: Self-Reporting Questionnaire; valor p: teste Quiquadrado de Pearson.

Fonte: Elaborado pelos autores.

peso entre as mulheres nesta pesquisa. Este resultado é similar aos obtidos em dois estudos nacionais realizados com dados secundários do VIGITEL $2006^{35}$ e $2008^{36}$, bem como, em estudo nos Estados Unidos com dados de 20 anos obtidos do National Longitudinal Study of Youth ${ }^{37}$. Pro- vavelmente os indivíduos que têm uma situação conjugal estável são mais propensos a ter uma companhia nas refeições e podem, portanto, ter o hábito de comer mais regularmente e/ou alimentos mais calóricos, propiciando o aumento de peso. Outra possível explicação é que casais com 
Tabela 3. Razão de Prevalência (RP) para o excesso de peso em mulheres dos 15 aos 49 anos, segundo variáveis sociodemográficas e reprodutivas. Recife, Brasil, 2017.

\begin{tabular}{|c|c|c|c|c|c|}
\hline Variáveis & RP Bruta & IC95\% & RP Ajustada & IC95\% & Valor $\mathrm{p}$ \\
\hline \multicolumn{6}{|l|}{ Bloco I } \\
\hline \multicolumn{6}{|l|}{ Classe Econômica } \\
\hline $\mathrm{D}, \mathrm{E}$ & 1,00 & & 1,00 & & \\
\hline $\mathrm{C} 2$ & 1,11 & $0,97-1,27$ & 1,11 & $0,98-1,27$ & 0,083 \\
\hline $\mathrm{B} 1, \mathrm{~B} 2, \mathrm{C} 1$ & 1,12 & $0,97-1,30$ & 1,09 & $0,94-1,25$ & 0,241 \\
\hline \multicolumn{6}{|l|}{ Faixa etária (anos) } \\
\hline $15-19$ & 1,00 & & 1,00 & & \\
\hline $20-35$ & 1,62 & $1,18-1,22$ & 1,50 & $1,09-2,07$ & 0,013 \\
\hline $36-49$ & 1,97 & $1,44-2,49$ & 1,83 & $1,33-2,51$ & $<0,001$ \\
\hline \multicolumn{6}{|l|}{ Cor da pele } \\
\hline Parda, Amarela e Indígena & 1,00 & & 1,00 & & \\
\hline Preta & 1,20 & $1,03-1,39$ & 1,16 & $1,02-1,38$ & 0,041 \\
\hline Branca & 1,21 & $1,07-1,37$ & 1,17 & $1,03-1,30$ & 0,013 \\
\hline \multicolumn{6}{|l|}{ Situação conjugal } \\
\hline Sem companheiro & 1,00 & & 1,00 & & \\
\hline Com companheiro & 1,23 & $1,11-1,37$ & 1,14 & $1,03-1,27$ & 0,019 \\
\hline \multicolumn{6}{|l|}{ Bloco II } \\
\hline \multicolumn{6}{|l|}{ Idade da Menarca (anos) } \\
\hline$\geq 12$ & 1,00 & & 1,00 & & \\
\hline$<12$ & 1,15 & $1,02-1,29$ & 1,17 & $1,05-1,31$ & 0,005 \\
\hline \multicolumn{6}{|l|}{ Número de gestações } \\
\hline Nulípara & 1,00 & & 1,00 & & \\
\hline $1-2$ & 1,29 & $1,03-1,62$ & 1,09 & $0,87-1,37$ & 0,506 \\
\hline$\geq 3$ & 1,59 & $1,28-1,98$ & 1,26 & $1,00-1,59$ & 0,043 \\
\hline
\end{tabular}

Fonte: Elaborado pelos autores.

um relacionamento estável, em geral têm menor preocupação com o seu peso corporal, por não estarem em busca de um companheiro ${ }^{38}$.

Em relação à idade quando do primeiro episódio menstrual, verificamos uma maior prevalência de excesso de peso entre aquelas que tiveram menarca precoce. Este resultado também é apontado em outros estudos transversais, no Brasil $^{7,32}$ e no Japão ${ }^{39}$, indicando ser a menarca precoce um fator que contribui para maior prevalência do sobrepeso e obesidade na vida adulta. Os resultados de outros estudos no nordeste brasileiro sugerem a menarca precoce como a precursora do excesso de peso em mulheres, nos estados do Ceará ${ }^{32}$ e Pernambuco ${ }^{19}$, assim como, nas mulheres de Minas Gerais, as quais tiveram chance três vezes maior de apresentar obesida$\mathrm{de}^{12}$. Entretanto, tem se questionado se a menarca precoce seria causa ou consequência do excesso de peso. Neste sentido, estudos longitudinais indicaram que a antecipação da menstruação seria decorrente de uma maior quantidade de tecido adiposo durante a infância, a qual desencadearia a produção do hormônio estrógeno, a partir de precursores andrógenos da adrenal e, desta forma, anteciparia a maturação sexual ${ }^{40,41}$.

$\mathrm{Na}$ presente investigação, o número de gestações influenciou a ocorrência do excesso de peso, achado este também observado em outros estudos, sendo apontado como um dos fatores de risco para a obesidade em mulheres ${ }^{12,42,43}$. Esse resultado é secundário à ingestão energética excessiva que influencia o ganho ponderal durante o período gestacional e a retenção de peso no pós-parto ${ }^{43-46}$, o qual pode perdurar ao longo da $\operatorname{vida}^{47}$, principalmente entre as mulheres com maior número de filhos ${ }^{42}$.

Os fatores socioeconômicos, como classe econômica e escolaridade, não se associaram ao excesso de peso nesta pesquisa, possivelmente por se tratar de uma comunidade de baixa renda. Este resultado difere do observado em outros estudos, realizados com maior representatividade dos estratos socioeconômicos, que demonstraram uma 
tendência de maior prevalência na população de baixa escolaridade e menor poder aquisitivo ${ }^{5,32,33}$.

Destaca-se como ponto positivo do estudo, o cuidado metodológico relacionado à seleção da amostra através de amostragem aleatória simples, minimizando assim o viés de seleção. Temos como limitações a impossibilidade de inferir relações de causalidade pela característica de transversalidade do estudo, o que pode gerar o viés de causalidade reversa, além do viés de memória inerente aos estudos com coleta retrospectiva das informações.

\section{Conclusões}

A prevalência do excesso de peso atingiu dois terços da população estudada em uma comunidade urbana de baixa renda, no Nordeste do Brasil. Foram identificados como fatores associados as características demográficas (idade cronológica, cor da pele, conviver com o companheiro) e reprodutivas (idade precoce da menarca e maior número de gestações). Esses resultados são simi- lares aos encontrados em amostras representativas para a região e o país, demonstrando a pandemia de excesso de peso.

Dentre os fatores associados, a multiparidade foi o único fator identificado passível de modificação, o que reforça a necessidade de destacar a importância dos serviços de pré-natal e planejamento familiar na prevenção e controle do excesso de peso, levando-se em consideração que uma parcela considerável de mulheres com esse problema nutricional é usuária desses serviços.

A redução da prevalência do excesso de peso contribui para o controle de doenças crônicas, destacando-se a hipertensão arterial e o diabetes mellitus tipo 2. Como o presente estudo identificou prevalências mais elevadas do excesso de peso com o avançar da idade cronológica, enfatizamos a importância de iniciar educação em saúde sobre hábitos alimentares saudáveis e ações de incentivo à prática de exercícios físicos regulares em faixas etárias mais jovens da população, como forma de propiciar mudanças de comportamento que favoreçam a redução do excesso de peso.

\section{Colaboradores}

L Dinegri e MC Lima participaram de todas as etapas da pesquisa e elaboração do artigo. HVD Santos e PIC Lira contribuíram com a interpretação dos dados e redação final do manuscrito. $\mathrm{M}$ Batista Filho, SH Eickmann e PC Cabral participaram da redação e revisão final do manuscrito.

\section{Agradecimentos}

Agradecemos à Fundação de Amparo à Ciência e Tecnologia (FACEPE) pelo financiamento a pesquisa, ao Conselho Nacional de Desenvolvimento Científico e Tecnológico (CNPq), pela concessão das bolsas de produtividade a $\mathrm{M} \mathrm{Ba-}$ tista Filho, MC Lima e PIC Lira e a Coordenação de Aperfeiçoamento de Pessoal de Nível Superior (CAPES) pela concessão da bolsa de Mestrado à L Dinegri. 


\section{Referências}

1. World Health Organization (WHO). Global status report on noncommunicable diseases 2014. Genebra: WHO; 2014.

2. World Health Organization (WHO). Prevalence of overweight among adults, ages 18+, 1975-2016 (age standardized estimate) Both sexes: 2016 [Internet]. [acessado $2018 \mathrm{dez} 23$ ]. Disponível em: http://gamapserver.who.int/gho/interactive_charts/ncd/risk_factors/overweight/atlas.html.

3. World Health Organization (WHO). Prevalence of overweight, ages 18+, 2016 (age standardized estimate) female [Internet]. [acessado $2018 \mathrm{dez} 23$ ]. Disponível em: http://gamapserver.who.int/mapLibrary/Files/ Maps/Global_Overweight_2016_Female.png.

4. Instituto Brasileiro de Geografia e Estatística (IBGE). Estudo Nacional de Despesa Familiar - ENDEF. Dados preliminares. Consumo alimentar, antropometria. Rio de Janeiro: IBGE; 1977.

5. Brasil. Ministério da Saúde (MS). Secretaria de Vigilância em Saúde. Departamento de Vigilância de Doenças e Agravos não Transmissíveis e Promoção da Saúde. Vigitel Brasil 2017: vigilância de fatores de risco e proteção para doenças crônicas por inquérito telefônico: estimativas sobre frequência e distribuição sociodemográfica de fatores de risco e proteção para doenças crônicas nas capitais dos 26 estados brasileiros e no Distrito Federal em 2017. Brasília: MS; 2018.

6. Costa EC, Lira PIC, Oliveira JS, Menezes RCE, Tavares FCLP, Batista Filho M. Evolução do excesso de peso e fatores associados em mulheres de 10 a 49 anos em Pernambuco, Nordeste, Brasil. Rev Nutr 2014; 27(5):513-524.

7. Andrade RG, Chaves OC, Costa DAS, Andrade ACS, Bispo S, Felicissimo MF, Friche AAL, Proietti FA, Xavier CC, Caiaffa WT. Overweight in men and women among urban area residents: individual factors and socioeconomic context. Cad Saude Publica 2015; 31(S11):148-158.

8. Peña M, Bacallao J. Obesity and Poverty a New Public Health Challenge. Washington, D.C.: PAHO; 2000.

9. Bezerra IN, Sichieri R. Sobrepeso e Obesidade: Um Problema de Saúde Pública. In: Taddei JAAC, Lang RMF, Logo-Silva G, Toloni MHA. Nutrição em Saúde Pública. Rio de Janeiro: Rubio; 2011.

10. Wanderley EN, Ferreira VA. Obesidade: uma perspectiva plural. Cien Saude Colet 2010; 5(1):185-194.

11. Kac G, Velasquez-Melendez G, Valente JG. Menarca, gravidez precoce e obesidade em mulheres brasileiras selecionadas em um Centro de Saúde de Belo Horizonte, Minas Gerais, Brasil. Cad Saude Publica 2003; 6(S1):S111-S118.

12. Teichmann L, Olinto MTA, Costa JSD, Ziegler D. Fatores de risco associados ao sobrepeso e a obesidade em mulheres de São Leopoldo, RS. Rev Bras Epidemiol 2006; 9(3):360-367.

13. Nast M, Oliveira A, Rauber F, Vitolo MR. Ganho de peso excessivo na gestação é fator de risco para o excesso de peso em mulheres. Rev Bras Ginecol Obstet 2013; 35(1):536-540.
14. Forte CC, Bernardi JR, Goldani MZ, Bosa VL. Relação entre a retenção de peso nos primeiros três meses pós -parto com ganho de peso e ingestão alimentar durante a gestação. Rev Bras Saude Matern Infant 2015; 15(3):279-287.

15. Ferreira VA, Magalhães R. Obesidade e pobreza: o aparente paradoxo. Um estudo com mulheres da Favela da Rocinha, Rio de Janeiro, Brasil. Cad Saude Publica 2005; 21(6):1976-1800.

16. Ferreira VA, Magalhães R. O corpo cúmplice da vida: considerações a partir dos depoimentos de mulheres obesas de uma favela carioca. Cien Saude Colet 2006; 11(2):483-490.

17. Ferreira VA, Silva AE, Rodrigues CAA, Nunes NLA, Vigato TC, Magalhães R. Desigualdade, pobreza e obesidade. Cien Saude Colet 2010; 15(1):1423-1432.

18. Prefeitura de Recife. Caracterização socioeconômica da comunidade dos Coelhos, Recife-PE [Internet]. [acessado 2018 jun 09]. Disponível em: http://www2.recife. pe.gov.br/servico/coelhos.

19. Pinheiro MM, Oliveira JS, Leal VS, Lira PIC Souza NP, Campos FACS. Prevalência do excesso de peso e fatores associados em mulheres em idade reprodutiva no Nordeste do Brasil. Rev Nutr 2016; 29(5):679-689.

20. Mari JJ, Williams PA. Validity Study of a Psychiatric Screening Questionnaire (SRQ-20) in Primary Care in the city of São Paulo. Br J Psychiatry 1986; 148:2326.

21. Lohman TG, Roche AF, Martorell R. Anthropometric standardization reference manual. Toronto: Abridged; 1991.

22. World Health Organization (WHO). AnthroPlus for personal computers. Manual: Software for assessing growth of the world's children and adolescents. Genebra: WHO; 2009.

23. World Health Organization (WHO). Physical status: The use and interpretation of anthropometry. WHO Technical Report Series No. 854. Genebra: WHO; 1995.

24. Associação Brasileira de Empresas de Pesquisa (ABEP). Critério de classificação econômica Brasil. São Paulo, 2014 [Internet]. [acessado 2018 nov 26]. Disponível em: http://www.abep.org/novo/default.aspx.

25. World Health Organization (WHO). A User's guide to the self reporting questionnaire ( $S Q R$ /compiled by Beusenberg M, Orley J) [Internet]. Genebra: WHO; 1994. [acessado 2018 jun 17]. Disponível em: http://www. who.int/iris/handle/10665/61113.

26. Brasil. Conselho Nacional De Saúde (CNS). Resolução no 196, de 10 de outubro de 1996. Diretrizes e normas regulamentadoras de pesquisa envolvendo seres humanos. Diário Oficial da União 1996;16 out.

27. Ferreira VA, Magalhães R. Obesidade entre os pobres no Brasil: a vulnerabilidade feminina. Cien Saude Colet 2011; 16(4):2279-2287.

28. Brasil. Instituto Brasileiro de Geografia e Estatística (IBGE). Pesquisa nacional de saúde: 2013: ciclos de vida: Brasil e grandes regiões/IBGE, Coordenação de Trabalho e Rendimento. Rio de Janeiro: IBGE; 2015. 
29. Gigante DP, França GVA, Sardinha LMV, Iser BPM, Meléndez GV. Temporal variation in the prevalence of weight and obesity excess in adults: Brazil, 2006 to 2009. Rev Bras Epidemiol 2011; 14(1):157-165.

30. Garmendia ML, Alonso FT, Kain J, Uauy R, Corvalan C. Alarming weight gain in women of a post-transitional country. Public Health Nutr 2013; 17(3):667673.

31. Kanguru L, McCaw-Binns A, Bell J, Yonger-Coleman $\mathrm{N}$, Wilks R, Hussein J. The burden of obesity in women of reproductive age and in pregnancy in a middle-income setting: A population based study from Jamaica. PLoS One 2017; 12(12):e0188677.

32. Correia LL, Silveira DMI, Silva AC, Campos JS, Machado MMT, Rocha HAL, Cunha AJLA, Lindsay AC. Prevalência e determinantes de obesidade e sobrepeso em mulheres em idade reprodutiva residentes na região semiárida do Brasil. Cien Saude Colet 2011; 16(1):133-145

33. Malta DC, Santos MA, Andrade SS, Oliveira TP, Stopa SR, Oliveira MM, Jaime P. Tendência temporal dos indicadores de excesso de peso em adultos nas capitais brasileiras, 2006-2013. Cien Saude Colet 2016; 21(4):1061-1069.

34. Pinho CPS, Diniz AS, Arruda IKG, Batista Filho M, Coelho PC, Sequeira LAS, Lira PIC. Prevalência e fatores associados à obesidade abdominal em indivíduos na faixa etária de 25 a 59 anos do Estado de Pernambuco, Brasil. Cad Saude Publica 2012; 29(2):313-324.

35. Gigante DP, Moura EC, Sardinha LMV. Prevalence of overweight and obesity and associated factors, Brazil, 2006. Rev Saude Publica 2009; 43(S2):83-89.

36. Sá NNB, Moura EC. Excesso de peso: determinantes sociodemográficos e comportamentais em adultos, Brasil, 2008. Cad Saude Publica 2011; 27(7):13801392.

37. Teachman J. Body weight, marital status, and changes in marital status. J Fam Issues 2016; 37(1):74-96.

38. Averett S, Sikora A, Argys L. For better for worse: Relationship status and body mass index. Econ Hum Biol 2008; 6(3):330-349.

39. Sumi A, Iwase M, Nakamura U, Fujii H, Ohkuma T, Ide H, Jodai-Kitamura T, Komorita Y, Yoshinari M, Kitazono T. Impact of age at menarche on obesity and glycemic control in Japanese patients with type 2 diabetes: Fukuoka Diabetes Registry. J Diabetes Investig 2018; 9(5):1216-1223.

40. Benedet J, Lopes AS, Adami F, Hinnig PF, Vasconcelos FAG. Association of sexual maturation with excess body weight and height in children and adolescents. BMC Pediatr 2014; 14:72.

41. Solorzano CMB, McCartney CR. Obesity and the pubertal transition in girls and boys. Reprod 2010; 140(3):399-410.

42. Castilho SD, Pinheiro CD, Bento CA, Barros-Filho AA, Cocetti M. Tendência secular da idade da menarca avaliada em relação ao índice de massa corporal. Arq Bras Endocrinol Metab 2012; 56(3):195-200.
43. Ferreira RAB, Benício MHD. Obesidade em mulheres brasileiras: associação com paridade e nível socioeconômico. Rev Panam Salud Publica 2015; 37(4/5):195200.

44. Jordão ISC, Kac G. Determinantes da retenção de peso pós-parto segundo a cor da pele em mulheres do Rio de Janeiro, Brasil. Rev Panam Salud Publica 2005; 18(6):403-411.

45. Harris HE, Ellison GTH. Do the changes in energy balance that occur during pregnancy predispose parous women to obesity. Nutr Res Rev 1997; 10(1):57-81.

46. Gunderson EP. Child bearing and obesity in women: weight before, during and after pregnancy. Obst Gynecol Clin North Am 2009; 36(2):317-334.

47. Siega-Riz, AM. A systematic review of outcomes of maternal weight gain according to the Institute of Medicine recommendations: birthweight, fetal growth, and postpartum weight retention. Am J Obstet Gynecol 2009; 201(4):339.e1-14.

Artigo apresentado em 06/02/2018

Aprovado em 16/11/2019

Versão final apresentada em 18/11/2019

Editores-chefes: Romeu Gomes, Antônio Augusto Moura da Silva 
